\title{
ULTRAVIOLET SPECTROPHOTOMETRY OF EARLY-TYPE STARS
}

\author{
K. NANDY, G. I. THOMPSON, and C. M. HUMPHRIES \\ Royal Observatory, Edinburgh, United Kingdom
}

\begin{abstract}
The ultraviolet spectra of B stars obtained with the sky scan telescope in the TD1 satellite have been used to obtain narrow-band magnitudes at several wavelengths. These photometric bands have an effective half-width of $100 \AA$. We have proposed a two-dimensional classification scheme based on ultraviolet colours, and some preliminary results are presented.
\end{abstract}

\section{Introduction}

Existing methods of classification of stars on MK systems are based on line intensities in the visible part of the spectrum. Since the energy distributions of hotter stars reach their Planck maximum in the far ultraviolet, a system of classification based on the observed ultraviolet colours will provide additional information on stellar physical parameters, e.g. effective temperature and surface gravity etc. The sky survey telescope in the TDI satellite gives stellar energy distributions on absolute scale at a resolution of $30 \AA$ and the wavelength coverage is from $1350 \AA$ to $2500 \AA$. In addition, there is a broad band measurement at $2740 \AA$. We have already obtained data for several thousands of early type stars, for many of which MK spectral types are not accurately known. In this paper, we shall propose a two-dimensional classification system based on ultraviolet colours.

The basis of classification given here is that after correcting for interstellar reddening the stars can be grouped according to their intrinsic flux distributions, which are determined primarily by their effective temperature (since the spectral types range considered here contains most of the energy of the hot stars). As we have shown in earlier papers (Humphries et al., 1975), luminous stars are increasingly deficient, with increasing $\frac{1}{\lambda}$, as compared to main sequence of same spectral types. Therefore, by proper choice of colours the stars can be separated according to their temperature and surface gravity.

\section{Observations and Deductions}

In order to achieve greater statistical accuracy, photometric bands of effective widths of $100 \AA$ have been derived from the observed spectra to give magnitudes at several wavelengths including $2500 \AA, 2190 \AA$ and $1490 \AA$. The main photometric error of the ultraviolet magnitudes obtained in this way is $\pm 0 .^{m} 04$ for stars brighter than $V=5^{m} \cdot 0$ rising to $\pm 0 !^{m} 12$ for fainter stars. The colour index $\left(m_{2190}-m_{2500}\right)$ has previously been shown (Humphries et al., 1973) to be a useful parameter for determining the amount of inter- 
stellar reddening without prior knowledge of the MK spectral type. The index changes from -0.5 for BOV to -0.3 for AOV but is very sensitive to interstellar reddening, the colour excess ratio $E_{2190}-2500 / E(B-V)$ being 2 . For a preliminary attempt to separate stars into natural groups having the same ultraviolet energy distributions we have first considered the sample of stars with only a small amount of reddening, as indicated by the observed colour $\left(m_{2190}-m_{2500}\right)$, assuming that this colour index has a mean value of -0.4 for B stars. This sample is restricted to stars with $E_{2190}-2500 \leqslant 0.3$ so that error due to uncertainties of interstellar extinction is small.

We have chosen the colour indices $\left(m_{2740}-V\right)$ and $\left(m_{1490}-m_{2740}\right)$ as earlier results indicated that the first primarily determines the colour-temperature while the second is sensitive to both temperature and luminosity. These colours have been corrected for interstellar extinction, using the colour index $\left(m_{2190}-m_{2500}\right)$ in conjunction with the main extinction law derived from our data (Nandy et al., 1975).

\section{Results and Discussion}

In Figure 1 we present the colour-colour diagram $\left(m_{1490}-m_{2740}\right)$ vs $\left(m_{2740}-V\right)_{0}$, where $V$ is the visual magnitude. Apart from $V$ magnitudes no other visual data have been used. It is found that points plotted in Figure 1 fall naturally into two groups; most of the observed points lie in a fairly narrow region in the upper part of the diagram (as denoted by crossed circles), and all of these are class III to V. However, a considerable number of points lie significantly lower (open circles), and all of these are found to be of luminosity class I and II. A sudden change in slope in the colour-colour diagram occurs at $\left(m_{2740}-V\right) \sim 0.5$. This is caused by the Planck maximum moving longward of $1490 \AA$ for cooler stars.

The separation between main sequence and supergiants is due to ultraviolet flux deficiency of luminous stars. It has been suggested (Humphries et al., 1975; Nandy and Schmidt, 1975) that the stars of lower surface gravity are cooler than the corresponding main-sequence stars of the same spectral type. In view of these results, for a twodimensional classification two extinction-free parameters $q_{1}$ and $q_{2}$ can be constructed, using $\left(m_{2190}-m_{2500}\right)$ as a reddening indicator, as follows:-

$$
\begin{aligned}
& q_{1}=\left(m_{2740}-V\right)-\frac{E_{2740}-V}{E_{2190-2500}}\left(m_{2190}-m_{2500}\right) \\
& q_{2}=\left(m_{1490}-m_{2740}\right)-\frac{E_{1490-2740}}{E_{2190-2500}}\left(m_{2190}-m_{2500}\right)
\end{aligned}
$$

The values of $\frac{E_{2740-V}}{E_{2190-2500}}$ and $\frac{E_{2490-2700}}{E_{2190--2500}}$ have been determined from the mean 


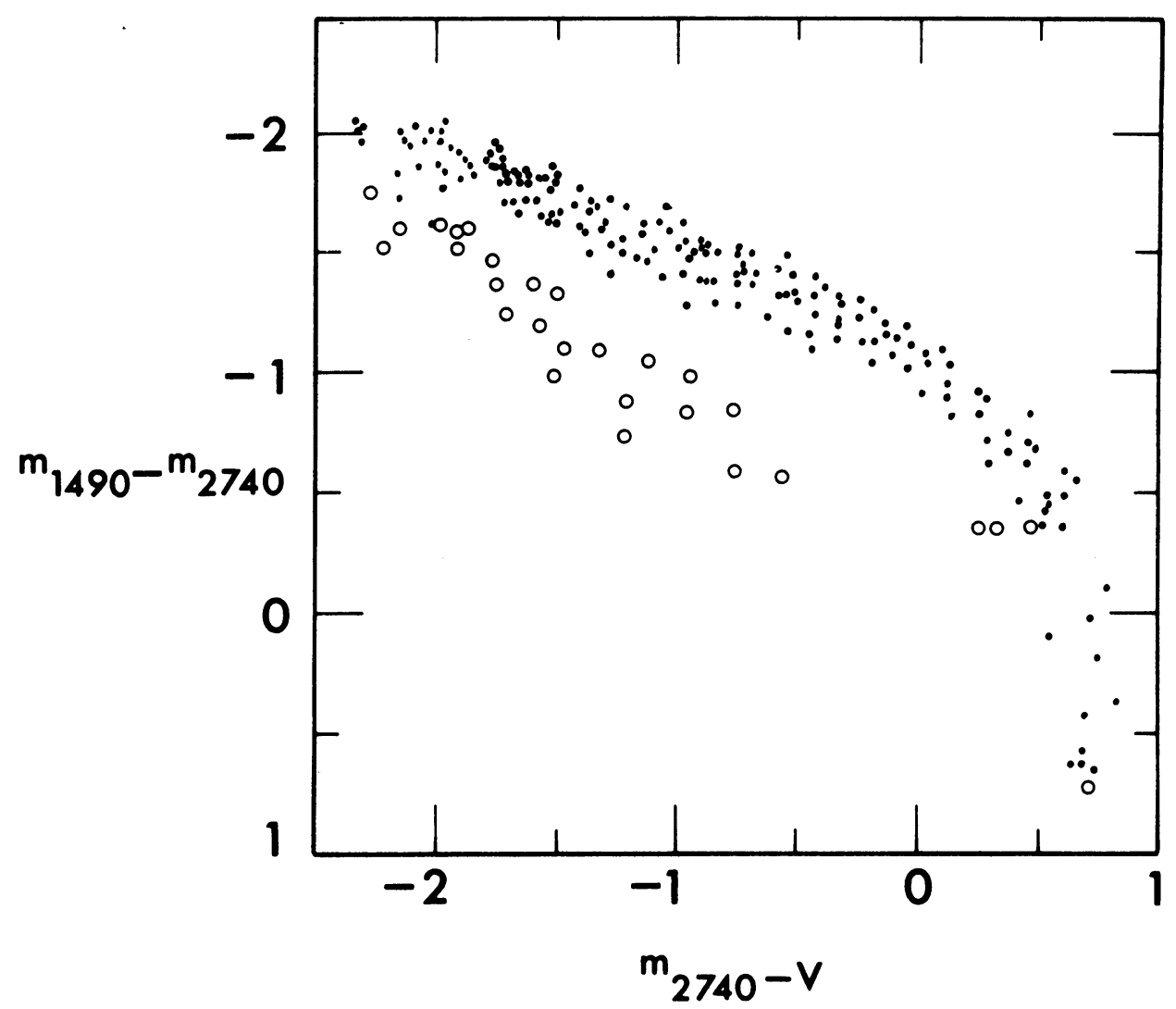

Fig. 1. The plot of $\left(m_{1490}-m_{2740}\right)$ vs $\left(m_{2740}-V\right)$. The stars of luminosity class I and II are shown by open circles; class III to $\mathrm{V}$ are indicated by dots.

extinction law derived from a sample of about a hundred reddened stars distributed in different galactic regions (Nandy et al., 1975). For these samples, no significant variation from the mean extinction law has been detected.

Figure 2 shows the plot of $q_{1}$ vs $q_{2}$ for a further batch of stars which show considerable amounts of reddening as, indicated by the colour index $\left(m_{2190} m_{2740}\right)$. Points plotted fall into two groups similar to the distribution as observed in Figure 1 . The unique division into groups I and II and III to V is as in Figure 1.

In order to establish the correspondence between the parameters $q_{1}$ and $q_{2}$, and MK spectral types, mean values of $q_{1}$ and $q_{2}$ have been determined from all the reddened and unreddened stars studied here; MK spectral types have been taken from the literature (Blanco et al., 1968). These values with the corresponding MK types are shown in Figure 2.

Be stars, however, probably do not fit this pattern. The $q_{1}$ and $q_{2}$ values of a sample of unreddened Be stars are shown in Figure 3 by circles. It appears that for Be stars of 


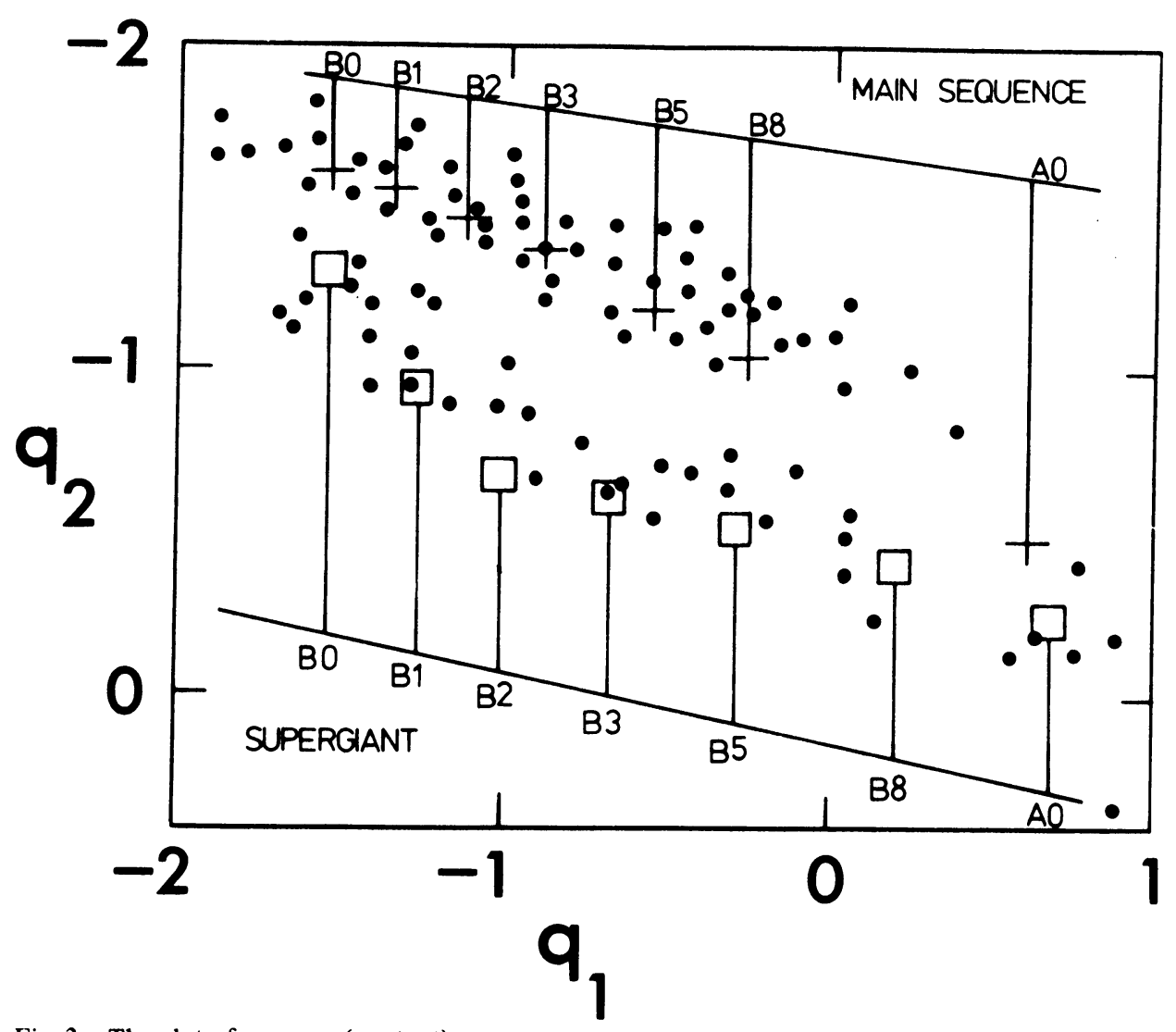

Fig. 2. The plot of $q_{1}$ vs $q_{2}$ (see text).

earlier types (B0-B3), $q_{1}$ and $q_{2}$ coincide with those of supergiants, while for later types they have the same values as main-sequence stars.

\section{References}

Humphries, C. M., Nandy, K., and Kontizas, E.: 1975, Astrophys. J. 195, 111.

Humphries, C. M., Nandy, K., and Thompson, G. I.: 1973, Monthly Notices Roy. Astron. Soc. 163, 1. Nandy, K. and Schmidt, E. G.: 1975, Astrophys. J. 198, 119.

Nandy, K., Thompson, G. I., Jamar, C., Monfils, A., and Wilson, R.: 1975, Astron. Astrophys. 44, 195.

\section{DISCUSSION}

Hauck: Have you observed sonie $\mathrm{Be}, \mathrm{Bp}$ or Ap stars?

Nandy: Yes, many of these stars have been observed, but I did not use them in this study since I wanted to stick to objects as normal as possible. Be stars do not fit in the diagram and are located between the class I and V sequences. 


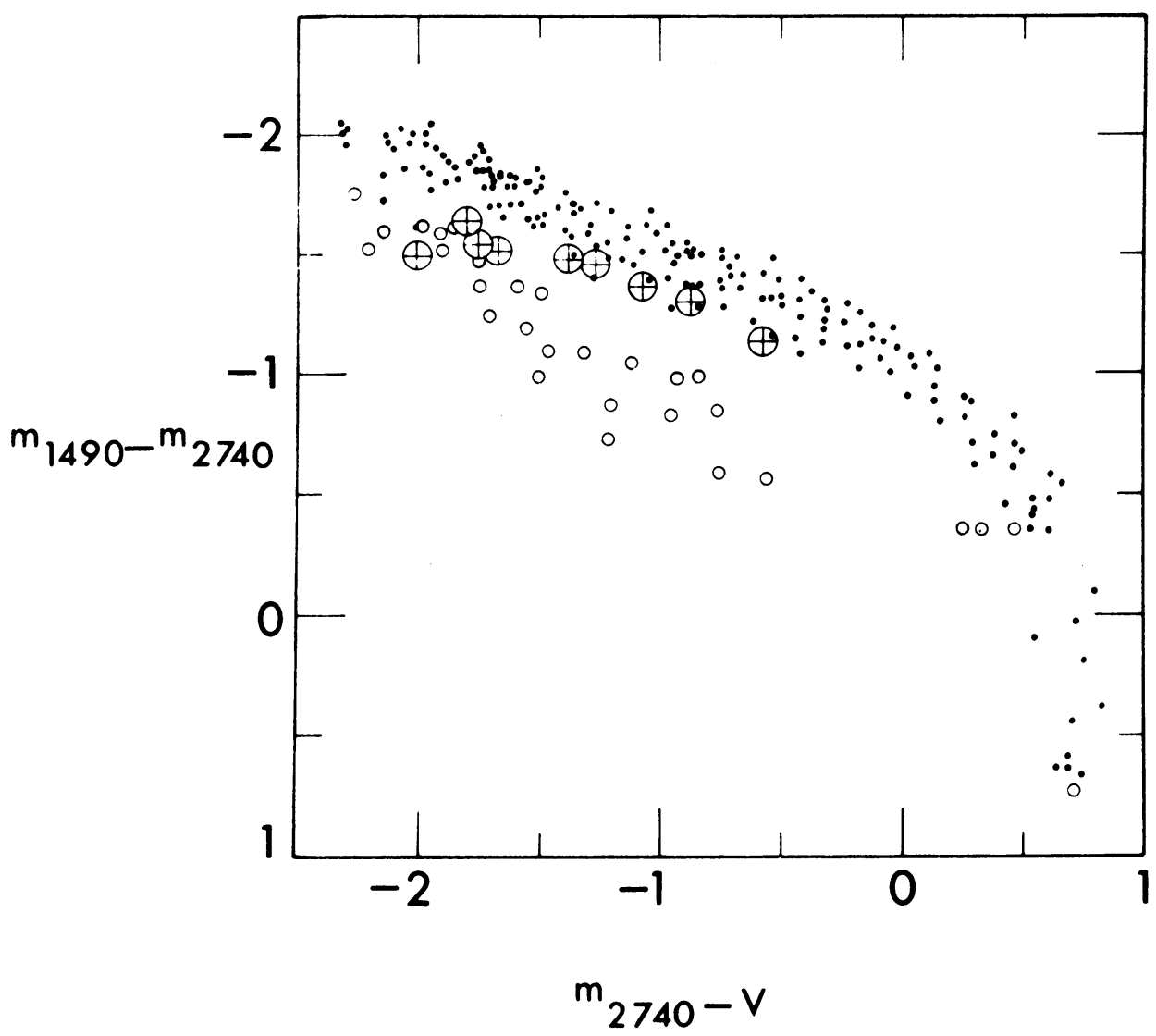

Fig. 3. Same as Figure 1. Be stars are represented by circles with cross.

Houziaux: I would like to mention that the details seen in the spectrum are not just spectral lines. A. Delcroix and myself are analysing these spectra using the spectral synthesis method and it appears that the spectral features result from the convolution of thousands of lines with the wide $(30 \AA)$ instrumental profile. The spectrum of B and A stars in the region 1500 to $2500 \AA$ is overcrowded with line absorptions.

Kandel: Since you mentioned the effects of rotation on the structure of stellar atmospheres on the first day, I wonder whether you have any evidence for such effects in your results, especially for B stars. Specifically, do you see any way to distinguish true slow rotators from pole-on fast rotators?

Nandy: We are, of course, interested in this problem, but so far we cannot extract information regarding rotation.

To the second question, the answer is no. 